\title{
Effect of Interleukin-1Beta (IL-1ß) on the Cortical Neurons Survival and Neurites Outgrowth
}

\author{
Ebtesam M. Abd-El-Basset \\ Department of Anatomy, Faculty of Medicine, Kuwait University, Kuwait city, Kuwait \\ Email: ebtesam@hsc.edu.kw
}

Received 3 December 2015; accepted 25 January 2016; published 29 January 2016

Copyright (c) 2016 by author and Scientific Research Publishing Inc.

This work is licensed under the Creative Commons Attribution International License (CC BY). http://creativecommons.org/licenses/by/4.0/

(c) (i) Open Access

\section{Abstract}

Insults to the brain are known to cause a myriad of downstream effects, including the release of cytokines by astrocytes and resultant reactive gliosis. The author has examined effect of cytokine IL-1 $\beta$ on the survival of cortical neurons using mouse astrocyte-neuron co-culture. Five groups were used. These were neurons alone (Group 1), neurons with added IL-1 $\beta$ (Group 2), neurons co-cultured with astrocytes (Group 3), neurons co-cultured with astrocytes that was pre-treated with IL-1 $\beta$ before co-culture (Group 4) and neurons co-cultured with astrocytes and IL-1 $\beta$ added (post-treated) (Group 5). In Group 1 only a few neurons grew and survived only for 5-6 days. In Group 2, it was observed that more neurons survived up to 11 days. Moreover, in Group 3, more neurons grew and survived up to 16-18 days. They had large cell bodies and many long neurites that formed anastomosing networks. In Group 4, few neurons survived up to 13 days, whereas in Group 5, the growth of neurons were affected but to a much lesser extent than Group 4 and survived up to 15 days. In addition, it was found that IL-1 $\beta$ stimulated the expression of glial fibrillary acidic protein (GFAP) by astrocytes. This study indicates that IL-1 $\beta$ affects the survival of cortical neurons and modulates the astrocytic support to neuronal survival and neurites outgrowth by acting directly on the astrocytes.

\section{Keywords}

Astrocytes, IL-1ß, Cell Culture, Neuronal Survival, Cytokines, Gliosis

\section{Introduction}

Astrocytes play an important role in neuronal migration and survival during the development of the central 
nervous system (CNS) [1] [2]. Astrocytes act also as important regulators of brain inflammation [3]. In addition, numerous studies have demonstrated the importance of astrocytes in a variety of neurophysiological processes such as the supply of energy metabolites, defense against oxidative stress or neurotransmitter reuptake and recycling [4]-[6].

Insults to the brain are known to cause a myriad of downstream effects, including the release of cytokines by astrocytes and resultant reactive gliosis [7] [8]. Cytokines are thought to be a major mediator of reactive gliosis [9]. IL- $1 \beta$ is a pro-inflammatory cytokine that exerts an important role in developing brains as a maintenance and growth-promoting factor [10] [11] and in migration of cortical neurons [12]. IL-1 $\beta$ is secreted by activated astroglia and microglia in brain, where it exerts a diverse range of activities on immune function and coordination of many aspects of the acute phase response to trauma and infection [8] [10]. Inflammation is the key hostdefense response to infection and injury but is also thought to be a major contributor to a diverse range of diseases. It is generally believed that the inflammatory processes stimulated by IL- $1 \beta$ is detrimental and can aggravate the primary damage caused by infections of the CNS [13]. The mechanisms by which IL- $1 \beta$ exerts its activities are not totally understood. The hypothesis of this study is that pro-inflammatory cytokine IL- $1 \beta$ acts on astrocytes to alter their chemical and physical properties, which in turn affects the survival of neurons. In this study the author have observed the direct effect of IL- $1 \beta$ on survival of cortical neurons and how IL- $1 \beta$-treated astrocytes modulate the survival of cortical neurons.

\section{Materials and Methods}

Animal care followed the recommendations of NIH Guidelines for Care and Use of Laboratory Animals and the Guide for the Care and Use of Laboratory Animals (Kuwait University-Faculty of Health Publication). All efforts were made to minimize animal suffering, to reduce the number of animals used, and to utilize alternatives to in vivo techniques.

\subsection{Astroglia Culture}

New-born Balb/c mice (from Health and Science Animal Laboratory, Kuwait University) were used. The brain was dissected and cells were disaggregated by gently forcing the neopallia through a nitex mesh of $75 \mu \mathrm{m}$. Cells were cultured both in culture flasks and on cover slips. A concentration of $5 \times 10^{4}$ and $5 \times 10^{6}$ nigrosine excluding cells were plated on $11 \times 22 \mathrm{~mm}$ glass cover slips in Petri dishes and on $75 \mathrm{~mm}$ flasks, respectively. The Petri dishes and the flasks were incubated with $3 \mathrm{ml}$ and $13 \mathrm{ml}$ of growth medium (MEM) (GibcoBRL) containing $5 \%$ horse serum (GibcoBRL) respectively. Some flasks were treated with 200 international units $(\mathrm{U}) / \mathrm{ml}$ of $\mathrm{IL}-1 \beta$ (Sigma) for 4 days. The cells were then incubated at $37^{\circ} \mathrm{C}$ in a humidified atmosphere with $5 \% \mathrm{CO}_{2}$ in air, the medium was changed every 2 days.

\subsection{Neuronal Culture}

The cerebral neuronal cultures were prepared according to the method reported in [14]. Balb/c mouse fetuses of embryonic day 15 were used. The brain was dissected to obtain the neopallia, and then disaggregated in $0.25 \%$ trypsin (GibcoBRL) in Pucks solution for $5 \mathrm{~min}$ at room temperature. Horse serum was added to stop the action of trypsin. The suspension was then centrifuged at $1000 \mathrm{rpm}$ for $5 \mathrm{~min}$. The pellet of cells was suspended in MEM containing $30 \mathrm{mM}$ glucose and further disaggregated by passing through a $75-\mu \mathrm{m}$ nitex mesh. These cells were then plated in 60-mm Petri dish containing $11 \times 22 \mathrm{~mm}$ cover slips coated with Poly-L-lysine(Sigma) (Group 1), then incubated in freshly prepared MEM containing $5 \%$ horse serum and $30 \mathrm{mM}$ glucose(Sigma). Some neuronal cultures were treated with $200 \mathrm{U} / \mathrm{ml}$ of IL-1 $\beta$ for 4 days (Group 2).

\subsection{Neuron-Astroglia Co-Culture}

Astroglia cultures were prepared as described above and then maintained for 10 days till a monolayer of cells were obtained. Neuronal cells obtained by the above procedure were seeded over this monolayer of astrocytes [15]. After 15 min of incubation the culture was rinsed and incubated with MEM containing 5\% horse serum and $30 \mathrm{mM}$ glucose (Group 3). Some astroglia were treated with $200 \mathrm{U} / \mathrm{ml} \mathrm{IL-1 \beta}$ for 4 days before adding the neurons (pre-treated) (Group 4), whereas, some co-cultures were treated with $200 \mathrm{U} / \mathrm{ml}$ IL-1 $\beta$ for 4 days (post-treated) (Group 5). 


\subsection{Immunostaining for Neurons}

Cells grown on cover slips were fixed in methanol at $-20^{\circ} \mathrm{C}$ for $4 \mathrm{~min}$, and then treated with PBS containing 3\% hydrogen peroxide. The cells were incubated with mouse momoclonalanti-neurofilament (NF) (Sigma) diluted 1:50 for one hour. This was then incubated with biotinylated-anti-mouse IgG (1:200) for 45 min followed by incubation of extra-avidin peroxidase (1:20) (Sigma) for $45 \mathrm{~min}$. The color was developed using DAB solution for 5 - $10 \mathrm{~min}$, then the cells on coverslips were washed with distilled water followed by dehydration in ascending grades of alcohol and cleared in the xylene and mounted in DPX.

\subsection{Morphological Studies and Neurite Outgrowth Essay}

The morphological changes in neurons after $3,5,7,13,17,20$ days of cultures were examined by phase contrast microscopy. To assess the number of survived neurons, the number of neurons extending neurites longer than two cell body diameters was counted in 8 randomly chosen microscopic fields using a 20x objective lens. The fields were examined from the center of each coverslip inside the tissue culture dish. Three dishes were examined from each experiment. Three separate independent experiments were used. The length of the neurites from the above neurons was measured using an imaging analysis program (Image J, version 1.04b, Wayne Rasband, NIH). In each experiment, 50 - 60 neurites were measured.

\subsection{Polyacrylamide Gel Electrophoresis (PAGE) and Immunoblotting}

Flasks of IL- $1 \beta$ treated astrocytes for 4 days and control of non-treated astrocytes were washed three times in PBS, scraped off and collected in PBS. The cells were immediately sonicated and the protein concentration was determined according to the method reported in [16]. The sonicated cells were dissolved in 10\% SDS-containing sample buffer and boiled for $3 \mathrm{~min}$. The same amount of protein $(20 \mu \mathrm{g} / \mathrm{well})$ from each sample was analyzed electrophoretically in 10\% SDS-PAGE gel according to the method reported in [17]. The proteins in the gel were transferred to nitrocellulose sheets by a modification of the method reported in [18]. After transfer, the nitrocellulose sheets were incubated for $1 \mathrm{~h}$ with $3 \%$ bovine serum albumin (BSA) in PBS. The nitrocellulose sheets were then incubated overnight at room temperature with rabbit polyclonal antibody to GFAP, diluted 1:500 in PBS containing 3\% BSA, then followed by affinity-purified goat anti-rabbit IgG conjugated to horse-radish peroxidase. The color was developed using freshly prepared $0.05 \%$ 4-chlor-1-naphthol and $0.015 \%$ $\mathrm{H}_{2} \mathrm{O}_{2}$ in PBS. The reaction was stopped by washing in tap water. All the chemicals and materials for electrophoresis and immunobloting were purchased from BioRad (Missisauga, Ont, Canada). Immunoblots of five independent experiments were scanned using the Snapescan 1212 scanner and Adobe Photoshop 5.0 program. The relative levels of GFAP expression were determined by analyzing the pixel intensity of the bands using an imaging analysis program (Image J, version 1.04b, Wayne Rasband, NIH). The percentage of the protein expression was calculated in the following manner. The average background protein levels in the lane, excluding the bands, were first subtracted from both the control and treatment bands. The percentage of the increase in intensity (I) of IL- $1 \beta$ treated cells (T) compared to the control (C) was calculated as follows:

$$
\text { Percentage of increase in the intensity }=100 \times \frac{\text { Mean } I \text { of } T-\text { Mean } I \text { of } C}{\text { Mean } I \text { of } C}
$$

\subsection{Statistical analysis}

Experiments were performed at least three times on different cell preparations. The data were analyzed with one-way Analysis of Variance (ANOVA) which rejected the null hypothesis. P-values $<0.05$ were considered statistically significant. A post-hoc Bonferroni’s test was used to compare the five groups individually

\section{Results}

\subsection{The Effect of IL- $1 \beta$ on Neuronal Survival}

When neurons were cultured alone on Poly-L-lysine coated coverslips (Group 1), few of them survived up to 5 6 days (Figure 1(a) and Figure 1(b)). These neurons had small cell bodies and few short neurites. When these neurons were treated with IL-1 $\beta$ (Group 2) they survived up to 10 days (Figure 1(c)). 


\subsection{The Effect of Astrocytes on the Neuronal Survival}

When the same number of neurons was cultured on top of normal astrocytes (Group 3), more neurons grew and they made anastomosing networks. The neurons had larger cell bodies and extended long neurites (Figure 2(a)). These neurons were grouped together and their neurites formed anastomosing networks in culture in 13-days culture (Figure 2(b)). The neurons survived up to 16 - 18 days (Figure 2(c)).

\subsection{Effect of IL-1 $\beta$-Pre-Treated Astrocytes on the Neuronal Survival}

When the same number of neurons were cultured on top of IL- $1 \beta$ pre-treated astrocytes (Group 4), few neurons attached (Figure 3(a)), and survived only for 13 days and had small cell bodies and short neurites (Figure 3(b)). The neurites did not form bundles as compare to the neurons grew on the top of non-treated astrocytes in (Figure 2(b)).

\subsection{Effect of IL- $1 \beta$ on the Neuronal Survival in Astrocyte-Neuron Co-Culture (Post-Treated)}

When astrocyte-neuron co-culture were treated with IL- $1 \beta$ for 12 days (Group 5), the survival of the neurons
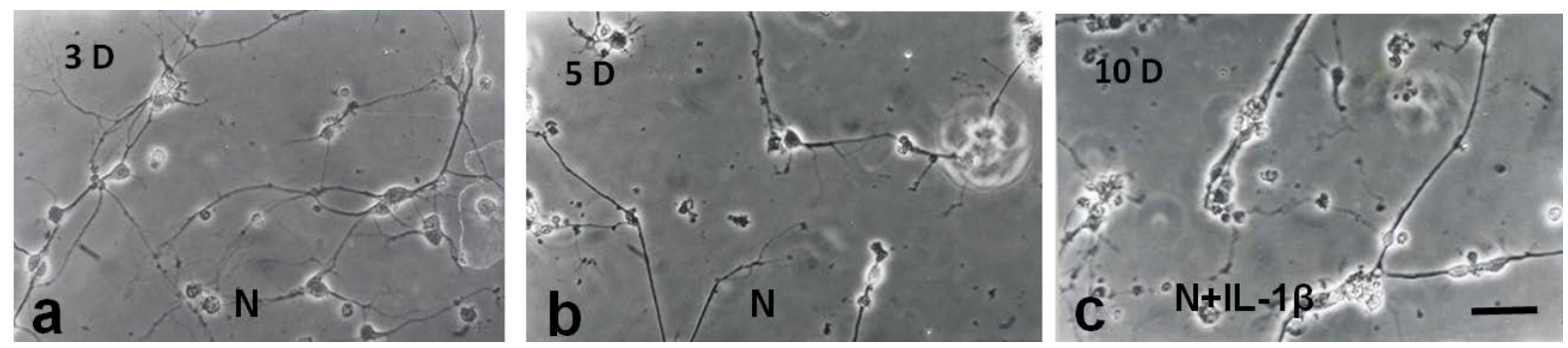

Figure 1. Phase contrast image of 3-day (a) and 5-day (b) old neuronal cultures growing alone without astrocytes (Group 1). Images show few numbers of neurons with small cell bodies and short neuritis. Phase contrast image of 10-days old neuronal cultures growing alone without astrocytes and treated with IL-1 $\beta$ (Group 2), the neurons survived up to 10 days (c). Scale bar is $50 \mu \mathrm{m}$.
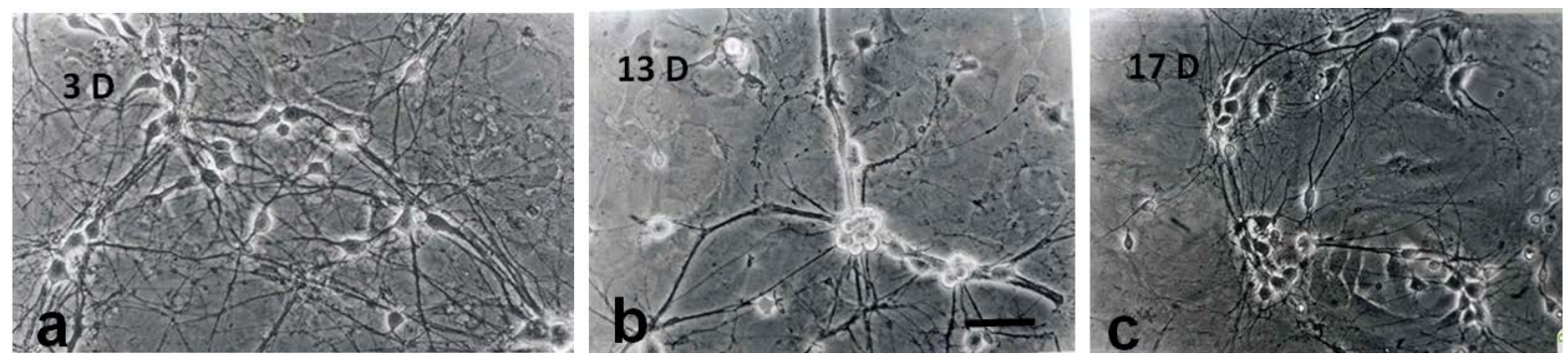

Figure 2. Phase contrast images of 3-day (a), 13-day (b), and 17-day (c) old neuronal cultures on the top of normal astrocytes (Group 3) showing many neurons grow and extend long neurites. Neuronal cultures on top of normal astrocytes of 13-days (b) showing many neurons form group together and the neurites form bundles. Scale bar is $50 \mu \mathrm{m}$.
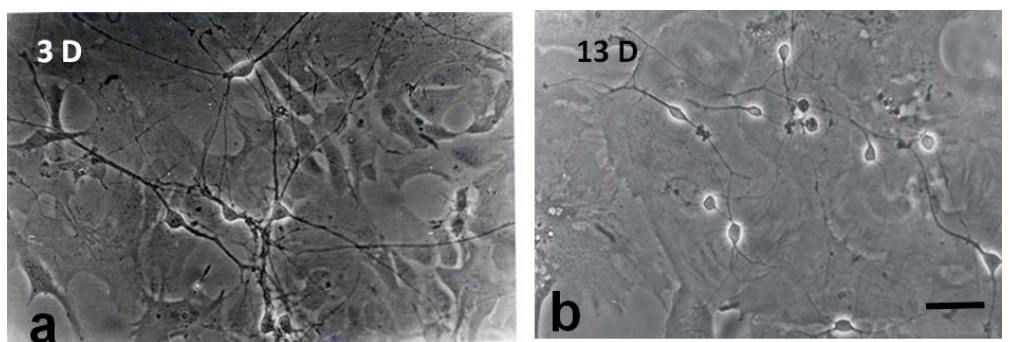

Figure 3. Phase contrast images of neurons on top of IL-1 $\beta$ pre-treated astrocytes of 3-day (a), and 13-day (b) old culture (Group 4) showing less survived neurons than when they grow on non-treated astrocytes. Scale bar is $50 \mu \mathrm{m}$. 
were affected but to a much lesser extent than the neuron grown on IL-1 $\beta$ pre-treated-astrocytes. The neurons survived up to 15 days (Figure 4).

\subsection{Immunostaining for Neurons}

When neurons and their neurites were stained for neurofilaments, many neurons and neurites were stained in 7-days old cultures grew on top of normal astrocyte (Group 3) compared to the neurons that grew on top of IL-1 $\beta$ pre-treated-astrocytes (Group 4) (Figure 5).

\subsection{The Neuronal Survival Essay}

In 3-day old culture, when neurons were cultured alone without astrocytes (Group 1) only few of them grew (10 \pm 1 ) and survived up to 5 - 6 days, while when they were treated with IL-1 $\beta$ (Group 2) they survived up to 10 days. When the same number of neurons was cultured on the top of astrocytes (Group 3), many neurons attached and survived $(75 \pm 4)$. However, when the same number of neurons were cultured on the top of IL- $1 \beta$ pre-treated astrocytes (Group 4) the number of survived neurons decreased to about ( $40 \pm 3$ ) neurons. In IL- $1 \beta$ post-treated co-culture (Group 5) the number of neurons attached is similar to the non-treated culture. In the older cultures (15, 17-days old) no neurons survived when cultured alone as well as when cultured on top of IL- $1 \beta$ pre-treated astrocytes. However, when they were cultured on top of normal astrocytes, about (35 \pm 5 ) neurons survived in the 13-days old culture, about (30 \pm 2 ) neurons survived in the 15-days old culture, and (25 \pm 2 ) neurons in the 17-days old culture (Figure 6). In post-treated co-culture about (10 \pm 1 ) neurons survived in 13-days and no neurons survived in 15-days old culture. There was a statistically significant increase in the number of neurons growing on the top of astrocytes (Group 3) (P-values $<0.001$ ) and longer period of survival (17 days) compared to the neurons growing alone (Group 1 ) or on the top of IL- $1 \beta$ pre-treated astrocytes (Group 4). There was no significant increase in the number of neurons alone (Group 1) or when they were treated with

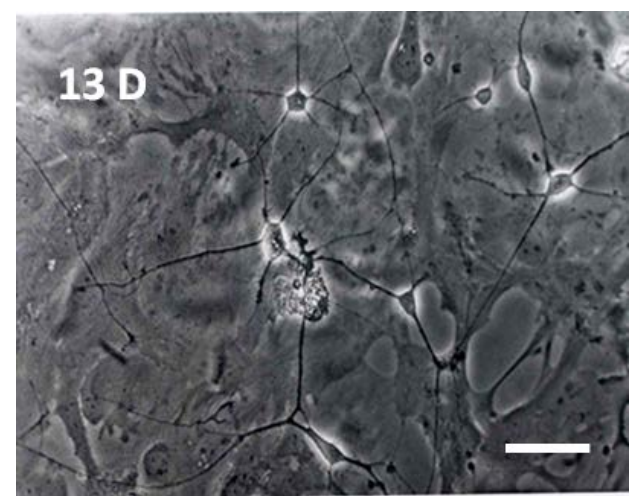

Figure 4. Phase contrast image of 13-day-old neuronal co-cultures (Group 5) showing only few neurons survived. Scale bar is $20 \mu \mathrm{m}$.
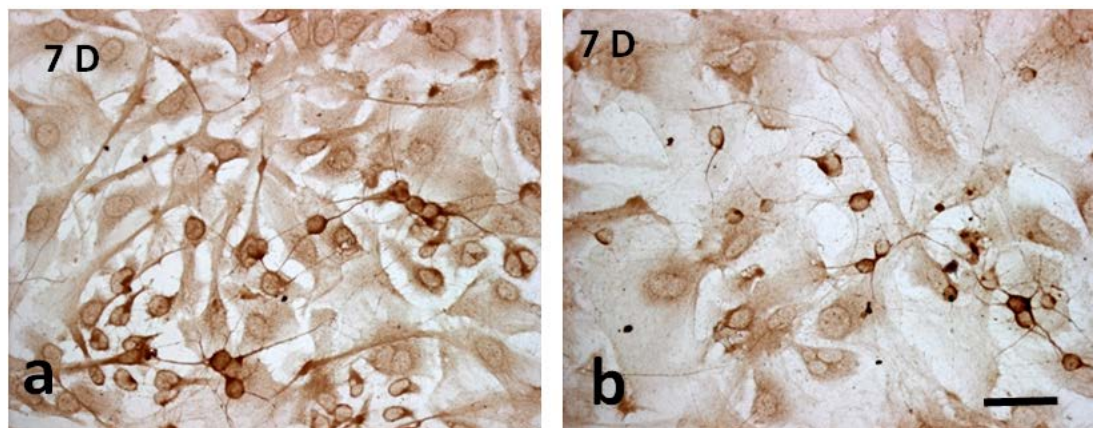

Figure 5. Immunocytochemistry staining with monoclonal antibody to neurofilaments of 7-day-old neuronal cultures on the top of normal astrocytes (a) (Group 3) and on pre-treated astrocytes with IL-1 $\beta$ (b) (Group 4) showing that many neurons with long neurites in figure (a) compare to figure (b). Scale bar is $50 \mu \mathrm{m}$. 


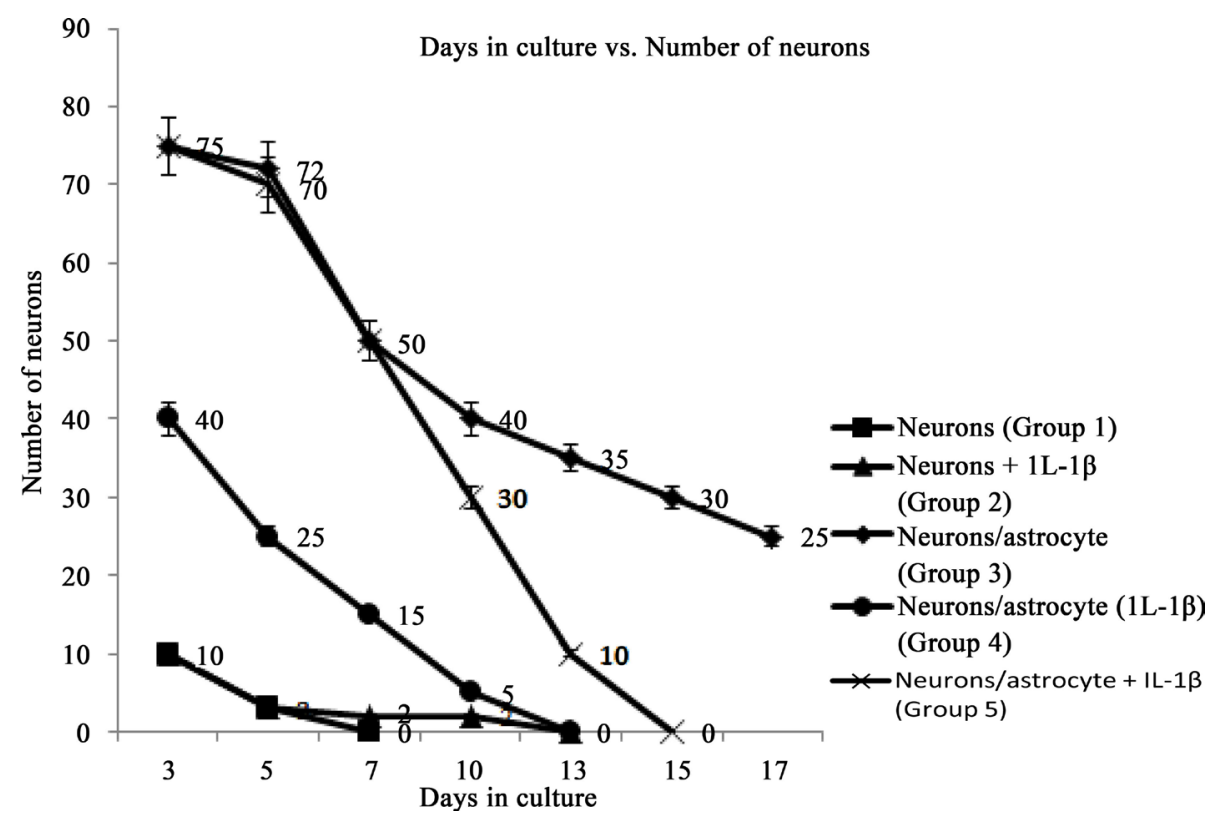

Figure 6. Graph showing number of neurons in five different conditions: Groups 1-5. Note the significant increase in the number of neurons growing on the top of astrocytes (Group 3) (P-values < 0.001) and longer period of survival (17 days) compared to the neuron growing alone (Group 1) or on the top of IL- $1 \beta$ pre-treated astrocytes (Group 4). There is no significant increase in the number of neurons alone when they were treated with IL- $1 \beta$ (Group2). Also there is no significant changes when the neuron on the top of normal astrocytes was post-treated with IL- $1 \beta$ (Group 5) compare to pre-treated astrocytes (Group 4).

IL-1 $\beta$ (Group 2). Also there were no significant changes when the neuron on the top of normal astrocytes (Group 3) compared to post-treated astrocytes (Group 5). A post-hoc Bonferroni's test was used to compare the three groups individually (Groups 1, 3 and 4), and showed that there was a significant difference between each of the three groups with a P-value of $<0.001$ for all groups.

\subsection{The Neurites Outgrowth Essay}

Measurement of the length of the neurites using the Image $\mathrm{J}$ analysis program showed that neurons cultured on the top of normal astrocytes (Group 3) have the longest neurites with progressive increases in the length with the days in culture compared to Group 1, Group 2, Group 4 or Group 5 (Figure 7). There was a significant difference between three groups, Group 1, Group 3 and Group 4 (P-values $<0.001$ ). A post-hoc Bonferroni's test was used to compare the three groups individually and showed that there was a significant difference between each of the three groups with a P-value of $<0.001$ for all groups. However, there was not a statistically significant difference in the length of neutites when comparing (Group 1) with (Group 2).

\subsection{Immunoblotting}

To determine the effect of IL- $1 \beta$ on the expression of GFAP in astrocytes, protein from 4 day IL- $1 \beta$ treated astrocytes and control astrocytes (non-treated) were separated by 10\% SDS-PAGE, transferred to nitrocellulose sheets and treated with antibody to GFAP (Figure 8). Quantification of the western blots of five independent experiments showed that the amount of GFAP in treated astrocytes increased to about $125 \%$ of control.

\section{Discussion}

This study shows that neuronal survival and neuritis outgrowth depends upon the presence of astrocytes. IL- $1 \beta$ induces gliosis in astrocytes which in turn inhibits neuronal survival. Although the direct effect of IL- $1 \beta$ on promoting neuronal survival is not significant, it can be explained by the cytokines ability to promote the use of growth factors by neurons [19]. The major effect of IL- $1 \beta$ is its effect on astrocytes. It inhibits the astrocytic support of neuronal survival and neuritis outgrowth. This modulation can be explained by astrogliosis induced in 


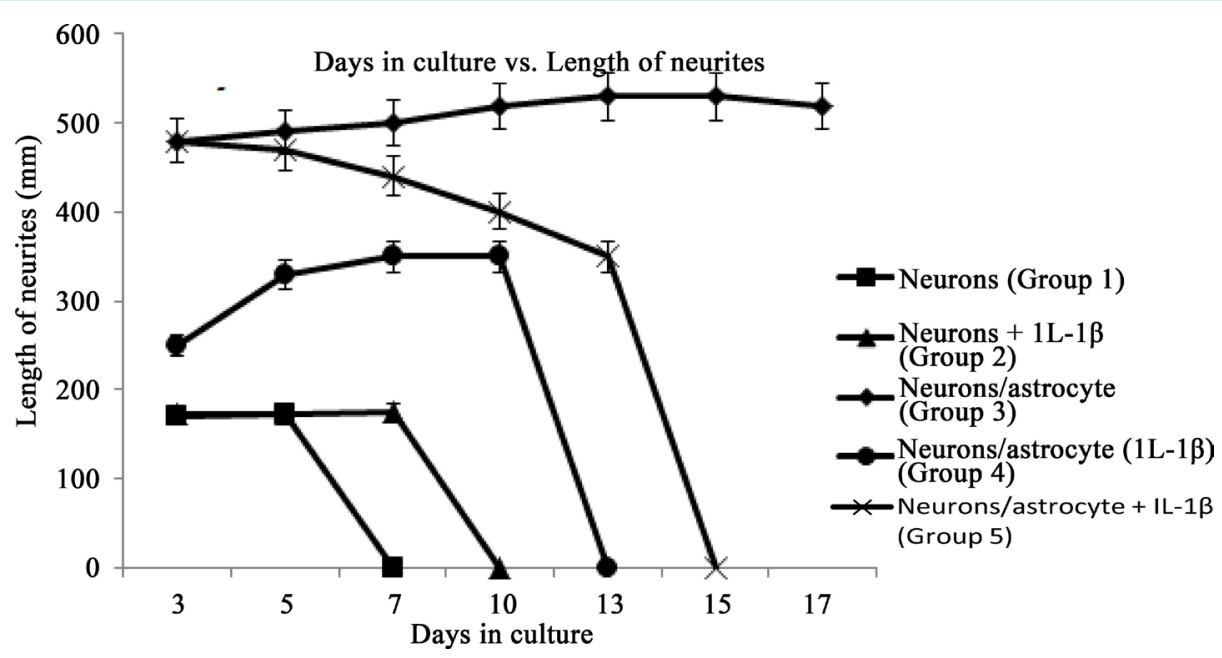

Figure 7. Graph showing the lengths of the neurites in 5 different conditions: (Groups 1-5) Note the significant increase lengths of the neurites of the neurons growing on top of astrocytes (Group 3) (P-values $<0.001$ ), and significant decrease lengths of neurites of neurons growing on the top of IL- $1 \beta$ pre-treated astrocytes (Group 4$)$ (P-values $<0.001$ ). There is no significant increase in length of neutites of neurons cultured alone and treated with IL-1 $\beta$ (Group 2) or in co-culture treated with IL-1 $\beta$ (Group 5). It should be notes that the sudden drop (to zero) in the length of the neurites in (Group 1 ) at day 7 or in (Group 2) at day 10 or in (Group 4) at day 13 and in (Group 5) at day 15 was due to the absence of the neurons and therefore the absence of neurites These lengths were magnified by $1220 \mathrm{x}$.

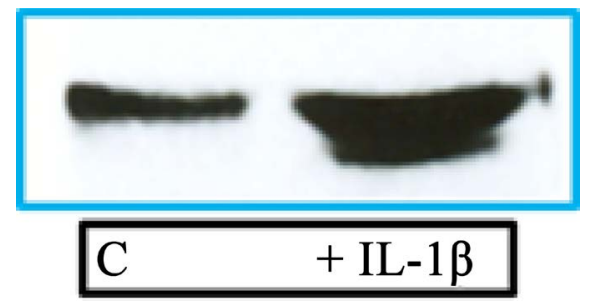

Figure 8. Immunoblotting of the transferred total protein extract of control astrocytes and 4 days IL- $1 \beta$-treated astrocytes stained with antibodies to GFAP. Note the increase level of GFAP in IL- $1 \beta$ treated astrocytes

IL-1 $\beta$ pre-treated astrocytes which may affect the chemical and physical properties of astrocytes so few neurons attached and survived. Previous studies have shown the role of IL- $1 \beta$ in induction of gliosis and neurodegeneration [20] [21]. Whether IL-1 $\beta$ regulates extracellular matrix (ECM) and adhesion molecules in astrogliosis is completely unclear, however, recent studies indicate the role of ECM in neuronal function and survival [22] [23].

The modifications of the astrocytic metabolism induced by IL-1 $\beta$ may modulate neuronal susceptibility to an exotoxic insult in neuron-astrocyte co-cultures. Together, these results suggest that IL- $1 \beta$ affects the metabolic profile of astrocytes, and that these changes have functional consequences for surrounding neurons. A previous study showed that IL-1 $\beta$ inhibited astroglia glutamic transport, which led to neurotoxicity [24].

Brain immune response is an essential process for protection against potentially deleterious threats such as infection, injury, cellular debris or abnormal protein aggregates. Although it is usually transient, persisting insults may give rise to chronic neuroinflammation which has been implicated in several neuropathology including Alzheimer's disease, Parkinson's disease, amyotrophic lateral sclerosis, multiple sclerosis, and stroke [25]. Neuroinflammation is a complex process, its overall effect is the result of a fine balance between a wide array of cytokines and growth factors, which may exert either neuroprotective or neurotoxic effects [26] [27]. Cytokines are major effectors in this fine balance as they have a dual function, potentially promoting or repressing neuroinflammation, hence their traditional classification as pro- or anti-inflammatory. Despite this classic denomination of cytokines, understanding their exact individual effect is far more complex as many of them interact with each other either synergistically or antagonistically and may additionally have pleiotropic effects [10]. Adding another level of complexity, different sets of cytokine receptors are expressed by the various cell types 
present in brain. Research over last 2 decades has revealed that IL-1 $\beta$ is an important mediator of neuronal injury and blocking the actions of IL- $1 \beta$ is beneficial in a number of experimental models of brain injury [28]. Mechanisms underlying the actions of IL- $1 \beta$ in brain injury remain unclear though increasing evidence indicates the cerebrovascular as a key target [29] [30]. The therapeutic use of anti-inflammatory agents may reduce CNS pathology induced by inflammatory responses. However, it would appear that the key to developing feasible anti-inflammatory drugs is to minimize the neurotoxic effects while promoting the beneficial and neurotrophic effects [31]-[33].

Many factors protect neurons through the inhibition of pro-inflammatory cytokines IL- $1 \beta$, such as erythropotein [34], fullerenols and glucosamine fluerences [35], omega-3 polyunsaturated fatty acids [36], and curcumin [37] [38]. In addition, recent evidence using a MAPKs inhibitor [39] and an IL-1 $\beta$ receptor antagonist [28] suggest that these may act as anti-inflammatory drugs.

In conclusion, this study indicates that IL- $1 \beta$ modulates the astrocytic support to neuronal survival and neurites outgrowth by acting directly on the astrocytes.

\section{Acknowledgements}

This work is supported by Kuwait University, Grant MA01/14. The author would like to thank Mrs. Amna Najem and Josily Joy for technical assistance.

\section{References}

[1] Furukawa, S., Furukawa, Y. and Satoyoshi, E. (1986) Synthesis and Secretion of Nerve Growth Factor by Mouse Astroglial Cells in Culture. Biochemical and Biophysical Research Communications, 136, 57-63. http://dx.doi.org/10.1016/0006-291X(86)90876-4

[2] Clarke, L.E. and Barres, B.A. (2013) Emerging Roles of Astrocytes in Neural Circuit Development. Nature Reviews Neuroscience, 14, 311-321. http://dx.doi.org/10.1038/nrn3484

[3] Farina, C., Aloisi, F. and Meinl, E. (2007) Astrocytes Are Active Players in Cerebral Innate Immunity. Trends in Immunology, 28, 138-145. http://dx.doi.org/10.1016/j.it.2007.01.005

[4] Belanger, M. and Magistretti, P.J. (2009) The Role of Astroglia in Neuroprotection. Dialogues in Clinical Neuroscience, 11, 281-295.

[5] Liddell, J.R., Robinson, S.R., Dringen, R. and Bishop, G.M. (2010) Astrocytes Retain Theirantioxidant Capacity into Advanced Old Age. Glia, 58, 1500-1509.

[6] Walls, A.B., Waagepetersen, H.S. and Bak, L.K. (2015) The Glutamine-Glutamate/GABA Cycle: function, Regional Differences in Glutamate and GABA Production and Effects of Interference with GABA Metabolism. Neurochemical Research, 40, 402-409. http://dx.doi.org/10.1007/s11064-014-1473-1

[7] Laping, N.J., Teter, B., Nichols, N.R., Rozovsky, I. and Finch, C.E. (1994) Glial Fibrillary Acidic Protein: Regulation by Hormones, Cytokines, and Growth Factors. Brain Pathology, 1, 259-275. http://dx.doi.org/10.1111/j.1750-3639.1994.tb00841.x

[8] Murray, K.N., Parry-Jones, A.R. and Allan, S.M. (2015) Interleukin-1 and Acute Brain Injury. Frontiers in Cellular Neuroscience, 9, 1-17. http://dx.doi.org/10.3389/fncel.2015.00018

[9] Little, A.R. and O’Callagha, J.P. (2001) Astrogliosis in Adult and Developing CNS: Is There a Role for Poinflammatory Cytokines? NeuroToxicology, 22, 607-618. http://dx.doi.org/10.1016/S0161-813X(01)00032-8

[10] John, G.R., Lee, S.C., Song, X., Rivieccio, M. and Brosnan, C.F. (2005) IL-1-Regulated Responses in Astrocytes: Relevance to Injury and Recovery. Glia, 49, 161-176. http://dx.doi.org/10.1002/glia.20109

[11] Boato, F., Hechler, D., Rosenberge, K., Lüdecke, D., Peters, E.M., Nitsch, R. and Hendrix, S. (2011) Interleukin-1 Beta and Neurotrophin-3 Synergistically Promote Neurite Growth in Vitro. Journal of Neuroinflammation, 8, 183. http://dx.doi.org/10.1186/1742-2094-8-183

[12] Ma, L., Li, X.W., Zhang, S.J., Yang, F., Zhu, G.-M., Yuan, X.-B. and Jiang, W. (2014) Interleukin-1 Beta Guides the Migration of Cortical Neurons. Journal of Neuroinflammation, 11, 114. http://dx.doi.org/10.1186/1742-2094-11-114

[13] Medel-Matus, J.S., Álvarez-Croda, D.M. and Martínez-Quiroz, J. (2014) IL-1 $\beta$ Increases Necrotic Neuronal Cell Death in the Developing Rat Hippocampus after Status Epilepticus by Activating Type I IL-1 Receptor (IL-1RI). International Journal of Developmental Neuroscience, 38, 232-240.

[14] Zhang, S. and Fedoroff, S. (1996) Neuron-Microglia Interactions in Vitro. Acta Neuropathologica, 91, 385-395. http://dx.doi.org/10.1007/s004010050440 
[15] Abd-El-Basset, E.M. (2013) Proinflammatory Tumor-Necrosis Factor-Alpha (TNF- $\alpha$ ) Inhibits Astrocytic Support of Neuronal Survival and Neurites Outgrowth. Advances in Bioscience and Biotechnology, 4, 73-80. http://dx.doi.org/10.4236/abb.2013.48A2010

[16] Bradford, M.M. (1976) A Rapid and Sensitive Method for Quantification of Microgram Quantities of Protein Utilizing the Principles of Protein-Dyebinding. Analytical Biochemistry, 72, 248-254. http://dx.doi.org/10.1016/0003-2697(76)90527-3

[17] Laemmli, U.K. (1970) Cleavage of Structural Proteins during Assembly of Head of the Bacteriophage T4. Nature, 227, 680-685. http://dx.doi.org/10.1038/227680a0

[18] Towbin, H., Staehlin, T. and Gordon, J. (1979) Electrophoretic Transfer of Proteins from Polyacrylamide Gels to Nitrocellulose Sheets: Procedure and Some Applications. Proceedings of the National Academy of Sciences of the United States of America, 76, 4350-4354. http://dx.doi.org/10.1073/pnas.76.9.4350

[19] Ho, A. and Blum, M. (1997) Regulation of Astroglial-Derived Dopaminergic Neurotrophic Factors by Interleukin-1 Beta in the Striatum of Young and Middle-Aged Mice. Experimental Neurology, 148, 348-359. http://dx.doi.org/10.1006/exnr.1997.6659

[20] Boutin, H., LeFeuvre, R.A. and Horai, R. (2001) Role of IL-1 $\alpha$ and IL-1 $\beta$ in Ischemic Brain Damage. The Journal of Neuroscience, 21, 5528-5534.

[21] Abd-El-Basset, E.M. and Abd-El-Barr, M.M. (2011) Effect of Interleukin-1 $\beta$ on the Expression of Actin Isoforms in Cultured Mouse Astroglia. The Anatomical Record, 294, 16-23. http://dx.doi.org/10.1002/ar.21303

[22] Summers, L., Kangwantas, K., Nguyen, L., Kielty, C. and Pinteaux, E. (2010) Adhesion to the Extracellular Matrix Is Required for Interleukin-1 Beta Actions Leading to Reactive Phenotype in Rat Astrocytes. Molecular and Cellular Neuroscience, 44, 272-281. http://dx.doi.org/10.1016/j.mcn.2010.03.013

[23] Summers, L., Kangwantas, K., Rodriguez-Grande, B., Denes, A., Penny, J., Kielty, C. and Pinteaux, E. (2013) Activation of Brain Endothelial Cells by Interleukin-1 Is Regulated by the Extracellular Matrix after Acute Brain Injury. Molecular and Cellular Neuroscience, 57, 93-103. http://dx.doi.org/10.1016/j.mcn.2013.10.007

[24] Prow, N.A. and Irani, D.N. (2008) The Inflammatory Cytokine, Interleukin-1 Beta, Mediates Loss of Astroglial Glutamate Transport and Drives Excitotoxic Motor Neuron Injury in the Spinal Cord during Acute Viral Encephalomyelitis. Journal of Neurochemistry, 105, 1276-1286. http://dx.doi.org/10.1111/j.1471-4159.2008.05230.x

[25] Allan, S.M. and Rothwell, N.J. (2003) Inflammation in Central Nervous System Injury. Philosophical Transactions of the Royal Society B: Biological Sciences, 358, 1669-1677. http://dx.doi.org/10.1098/rstb.2003.1358

[26] Ross, F.M., Allan, S.M., Rothwell, N.J. and Verkhratsky, A. (2003) A Dual Role for Interleukin-1 in LTP in Mouse Hippocampal Slices. Journal of Neuroimmunology, 144, 61-67. http://dx.doi.org/10.1016/j.jneuroim.2003.08.030

[27] Song, C., Zhang, Y. and Dong, Y. (2012) Acute and Subacute IL-1 $\beta$ Administrations Differentially Modulate Neuroimmune and Neurotrophic Systems: Possible Implications for Neuroprotection and Neurodegeneration. Journal of Neuroinflammation, 10, 59.

[28] Schizas, N., Andersson, B., Hilborn, J. and Hailer, N.P. (2014) Interleukin-1 Receptor Antagonist Promotes Survival of Ventral Horn Neurons and Suppresses Microglial Activation in Mouse Spinal Cord Slice Cultures. Journal of Neuroscience Research, 92, 1457-1465. http://dx.doi.org/10.1002/jnr.23429

[29] Murray, K.N., Girad, S., Holemes, W.M., Parkes, L.M., Williams, S.R., Parry-Jones, A.R. and Allan, S.M. (2014) Systemic Inflammation Impairs Tissue Reperfusion through Endothelin-Dependent Mechanisms in Cerebral Ischemia. Stroke, 45, 3412-3419. http://dx.doi.org/10.1161/STROKEAHA.114.006613

[30] Chapouly, C., Tadesse Argaw, A., Horng, S., Castro, K., Zhang, J., Asp, L., et al. (2015) Astrocytic TYMP and VEGFA Drive Blood-Brain Barrier Opening in Inflammatory Central Nervous System Lesions. Brain, 138, 1548-1567. http://dx.doi.org/10.1093/brain/awv077

[31] Kumar, A. and Loane, D.J. (2012) Neuroinflammation after Traumatic Brain Injury: Opportunities for Therapeutic Intervention. Brain, Behavior, and Immunity, 26, 1191-1201. http://dx.doi.org/10.1016/j.bbi.2012.06.008

[32] Finnie, J.W. (2013) Neuroinflammation: Beneficial and Detrimental Effects after Traumatic Brain Injury. Inflammopharmacology, 21, 309-320. http://dx.doi.org/10.1007/s10787-012-0164-2

[33] Ransohoff, R.M., Schafer, D., Vincent, A., Blachère, N.E. and Bar-Or, A. (2015) Neuroinflammation: Ways in Which the Immune System Affects the Brain. Neurotherapeutics, 12, 896-909. http://dx.doi.org/10.1007/s13311-015-0385-3

[34] Noh, M.Y., Cho, K.A., Kim, H., Kim, S.M. and Kim, S.H. (2014) Erythropoietin Modulates the Immune-Inflammatory Response of a SOD1 ${ }^{\text {G93A }}$ Transgenic Mouse Model of Amyotrophic Lateral Sclerosis (ALS). Neuroscience Letters, 574, 53-58. http://dx.doi.org/10.1016/j.neulet.2014.05.001

[35] Fluri, F., Grünstein, D., Cam, E., Ungethuem, U., Hatz, F., Schäfer, J., et al. (2015) Fullerenols and Glucosamine Fullerenes Reduce Infarct Volume and Cerebral Inflammation after Ischemic Stroke in Normotensive and Hypertensive 
Rats. Experimental Neurology, 265, 142-151. http://dx.doi.org/10.1016/j.expneurol.2015.01.005

[36] Zendedel, A., Habib, P., Dang, J., Lammerding, L., Hoffmann, S., Beyer, C. and Slowik, A. (2015) Omega-3 Polyunsaturated Fatty Acids Ameliorate Neuroinflammation and Mitigate Ischemic Stroke Damage through Interactions with Astrocytes and Microglia. Journal of Neuroimmunology, 278, 200-211.

http://dx.doi.org/10.1016/j.jneuroim.2014.11.007

[37] Li, Y., Li, J., Li, S., Li, Y., Wang, X., Liu, B., et al. (2015) Curcumin Attenuates Glutamate Neurotoxicity in the Hippocampus by Suppression of ER Stress-Associated TXNIP/NLRP3 Inflammasome Activation in a Manner Dependent on AMPK. Toxicology and Applied Pharmacology, 286, 53-63. http://dx.doi.org/10.1016/j.taap.2015.03.010

[38] Yuan, J., Zou, M., Xiang, X., Zhu, H., Chu, W., Liu, W., et al. (2015) Curcumin Improves Neural Function after Spinal Cord Injury by the Joint Inhibition of the Intracellular and Extracellular Components of Glial Scar. Journal of Surgical Research, 195, 235-245. http://dx.doi.org/10.1016/j.jss.2014.12.055

[39] Liu, X.W., Ji, E.F., He, P., Xing, R.X., Tian, B.X., Li, X.D., et al. (2014) Protective Effects of the p38 MAPK Inhibitor on NMDA-Induced Injury in Primary Cerebral Cortical Neurons. Molecular Medicine Reports, 10, 1942-1948. http://dx.doi.org/10.3892/mmr.2014.2402 